and describes manifestations and correlates of $\mathrm{MJ}$ and $\mathrm{CC}$ among participants.

Methods A cross sectional study using multistage cluster sampling was conducted among public high school students, aged 14-19 years, in two districts in Panama City from AugustNovember 2015 and June-August 2016. Participants selfadministered a questionnaire and gave biological samples. Those who acknowledged prior sexual activity (PSA) were tested for HIV and other STI (syphilis, chlamydia, gonorrhoea, trichomoniasis, M. genitalium, and hepatitis B surface antigen). Results Of the 1228 male and female participants, $57.7 \%$ of males and $58.5 \%$ of females reported PSA; $23.1 \%$ of participants reporting PSA had $\geq 1$ positive STI test. Positive STI tests or PSA were not correlated with MJ or CC. Manifestations of MJ were found in $19.6 \%$ of participants; correlates include low confidence in personal HIV knowledge $(\mathrm{AOR}=2.5,95 \% \mathrm{CI}: 1.3-5.0)$ and belief that HIV+ classmates should be excluded from school (CES) (AOR $=2.7$ 95\% CI: 1.6-4.6). Almost half $(48.8 \%)$ of participants answered affirmatively to 2-9 scenarios of transmission by CC. High confidence in personal HIV knowledge was protective for belief of transmission through $\mathrm{CC}(\mathrm{AOR}=0.6,95 \% \mathrm{CI}$ : 0.4-0.9); participants with belief in CES were more likely to believe in transmission by CC (AOR=2.0 95\% CI: 1.3-3.2).

Conclusion This study found high STI prevalence in public school adolescents from two districts in Panama City. Poor confidence in personal HIV knowledge and exclusion through CES were correlated with MJ of PLHIV, and belief in HIV transmission through CC. This study identifies the need for effective educational interventions to increase HIV transmission knowledge and decrease negative attitudes towards PLHIV.

Support: Funding for this study came from the Panamanian Ministry of Economics and Finance

\section{P3.09 LONGITUDINAL TRAJECTORIES OF SEXUAL RISK BEHAVIOUR IN MEN WHO HAVE SEX WITH MEN}

\begin{abstract}
${ }^{1,2} \mathrm{M}$ Basten, ${ }^{1} \mathrm{~J}$ Heijne, ${ }^{2,3} \mathrm{R}$ Geskus, ${ }^{1} \mathrm{C}$ den Daas, ${ }^{1,4} \mathrm{M}$ Kretzschmar, ${ }^{2} \mathrm{~A}$ Matser. ${ }^{1} \mathrm{C} e n t r e$ for infectious Diseases Control, National Institute for Public Health and the Environment (RIVM), Bilthoven, The Netherlands; ${ }^{2}$ Department of Infectious Diseases, Public Health Service Amsterdam, The Netherlands; ${ }^{3}$ Department of Clinical Epidemiology, Biostatistics and Bioinformatics, Academic Medical Centre, Amsterdam, The Netherlands; ${ }^{4}$ Julius Centre for Health Sciences and Primary Care, University Medical Centre Utrecht, Utrecht, The Netherlands
\end{abstract}

\subsection{6/sextrans-2017-053264.246}

Introduction Sexual behaviour changes during a person's life course. Insights in sexual careers of men who have sex with men (MSM) may optimise timing of HIV prevention methods. Our objectives were to develop a behavioural risk score for HIV seroconversion and study trajectories of sexual behaviour over time.

Methods Longitudinal data from the Amsterdam Cohort Studies on HIV and AIDS (ACS) were used. We included HIV-negative MSM who visited the ACS at least once between May 2007 and April $2016(\mathrm{n}=820)$. MSM were bi-annually tested for HIV and completed a questionnaire about their sexual behaviour in the preceding six months. The following behavioural variables were selected a priori: number of casual partners with insertive or receptive anal intercourse (AI), condom use during AI, number of condomless AI partners with unknown or positive HIV status, and AI during group sex. Using Poisson regression, we calculated a risk score for HIV seroconversion. This score was used in growth mixture modelling to examine trajectories of sexual behaviour since first male sexual contact.

Results During follow-up 49 MSM seroconverted. Predictors of seroconversion in multivariable analyses were number of casual partners with receptive AI (log transformed IRR: 1.63, 95\% CI: 1.20-2.22) and number of condomless insertive AI partners with unknown or positive HIV status (log transformed IRR: 1.73 , 95\% CI: 1.24-2.42). We identified 3 sexual risk behaviour trajectories: Decreasing high risk $(7 \%$ of MSM) with high risk behaviour at start of sexual career and decreasing risk over time; Increasing high risk (3\%) with low risk behaviour at start of sexual career and increasing risk over time; and Low risk (90\%) with relatively low risk throughout the career. MSM in the decreasing high risk trajectory were younger at first sexual contact than MSM in the Low risk trajectory.

Conclusion We identified 3 distinct sexual career trajectories. Increasing risk behaviour during the life course was found in a small group of MSM. The trajectories of these men might provide clues for time-tailored interventions.

\section{P3.10 HPV CLEARANCE IN A BRAZILIAN WOMEN LIVING WITH HIV COHORT}

${ }^{1,2,3}$ AGA Travassos, ${ }^{4}$ EM Netto, ${ }^{4} E$ Xavier-Souza, ${ }^{2,3}$ IP Nóbrega, ${ }^{3} \mathrm{KSAG}$ Brandão, ${ }^{2,3}$ PMA Silva, ${ }^{3} \mathrm{SA}$ Fernandes, ${ }^{3} \mathrm{~K}$ Abbehusen, ${ }^{4} \mathrm{M}$ Timbó, ${ }^{3} \mathrm{~T}$ Haguihara, ${ }^{5} \mathrm{FS}$ Ferreira, ${ }^{4}$ CA Brites. 'Universidade do Estado da Bahia - UNEB, Brazil; ${ }^{2}$ Brazilian Society for STD, Brazil; ${ }^{3}$ Centro Especializado em Diagnóstico, Assistência e Pesquisa - CEDAP, Brazil; ${ }^{4}$ Universidade Federal da Bahia - UFBA; ${ }^{5}$ Laboratório Central de Saúde Pública da Bahia LACEN/BA, Brazil

\subsection{6/sextrans-2017-053264.247}

Introduction Persistent infection with high-risk human papillomavirus (HR-HPV) is necessary for the development of precursor lesions and cervical cancer. HPV infection among women living with HIV/AIDS (WLHA) occurs more frequently, presents a higher parcel of persistent infections and an earlier progression to cancer. We aimed to evaluate the HR-HPV infection and clearance, and its association with HIV viral suppression, immunological response, and other risk factors among WLHA followed in a STD/HIV reference centre.

Methods This is a cohort study conducted at a reference centre for STD/AIDS in Northeastern Brazil from September 2013 to September 2015. Follow-up visits were conducted at 6 and 12 months from enrolment, where socio-epidemiological data was obtained through standardised form. Cervical samples were collected for conventional cytology and HPV DNA research (PCR COBAS Roche) in addition to blood samples for TCD4+ lymphocyte count and HIV viral load.

Results We prospectively evaluated 333 women. HR-HPV DNA prevalence was $39.6 \%$ during the follow-up. HPV-16 was present in $6.0 \%$, HPV-18 in 5.4\% and $37.8 \%$ WLHA had other HR-HPV $(31,33,35,39,45,51,52,56,58,59,66$ and 68). 